\title{
Educación alimentaria: salud y cohesión social
}

\author{
Food education: health and social cohesion
}

'Doctora en Antropología Social. Profesora agregada interina, Departamento de Antropología, Filosofía y Trabajo Social, Universitat Rovira i Virgili, Tarragona, España. $\triangle$ iD
RESUMEN Desde un abordaje teórico-reflexivo, este artículo interconecta los resultados de diversas investigaciones cualitativas con teorías del conflicto social y de la antropología médica, para investigar cómo la alimentación puede ser una herramienta de transformación social, en términos de salud, pero también de diálogo, respeto y convivencia de personas, grupos y comunidades. En este sentido, se presenta una primera aproximación a un nuevo enfoque teórico-metodológico sobre educación alimentaria. En este enfoque, la alimentación incorpora una perspectiva política, sociocultural y participativa que nos acerca a una comprensión innovadora del fenómeno: la alimentación como herramienta de análisis y diagnóstico de realidades socioalimentarias, pero también como instrumento de intervención educativo-sanitaria en la resolución de conflictos y en el fomento de sociedades más saludables en términos globales: nutricionales y también de igualdad y cohesión social.

PALABRAS CLAVES Educación Alimentaria; Antropología Médica; Participación Social; Política Alimentaria; Factores Culturales; Cambio Social.

\begin{abstract}
Using a theoretical-reflexive approach, this article connects the results of various qualitative studies in social conflict and medical anthropology, in order to investigate how food can be a tool for social transformation in terms of health but also in terms of the dialogue, respect and coexistence among people, groups and communities. In this sense the article presents a first approximation to a new theoretical and methodological approach to food education. In this approach, food adopts a political, sociocultural and participatory perspective that brings us closer to an innovative understanding of the phenomenon of food: not only as an analytic and diagnostic tool, but also as an instrument for health education interventions toward conflict resolution and the promotion of healthier societies overall - nutritionally, but also in terms of equality and social cohesion.
\end{abstract}

KEY WORDS Food Education; Medical Anthropology; Social Participation; Food Policy; Cultural Factors; Social Change. 


\section{INTRODUCCIÓN}

El interés creciente por la educación alimentaria de niños, niñas y adolescentes se ha visto representado por el diseño de numerosas estrategias políticas. Entre otras, tenemos la Estrategia Mundial sobre Régimen Alimentario, Actividad Física y Salud de la Organización Mundial de la Salud ${ }^{(1)}$; el Libro Blanco "Estrategia Europea sobre Problemas de Salud relacionados con la Alimentación, el Sobrepeso y la Obesidad"(2); la Estrategia para la Nutrición, Actividad Física y Prevención de la Obesidad ${ }^{(3)}$ y el Plan Cuídate $+2012^{(4)}$, en el Estado español. Asimismo, la Generalitat de Catalunya (el Gobierno Autonómico de Cataluña) publica, en forma recurrente, materiales pedagógicos dirigidos a las escuelas y a las familias con el propósito de promover una alimentación saludable.

No obstante, la mayoría de estas políticas sobre educación alimentaria no tienen en cuenta el contexto sociocultural en el que la comida se enmarca y el hecho de que lo que finalmente comen o no los niños y las niñas (la ingesta real) está profundamente influenciado por factores como el género; la etnia; las ideologías; el contexto educativo, normativo, organizativo y dinámico del espacio familiar, escolar o comunitario en el que se ingiere la comida; el rol que juega el placer, la comodidad y la salud; las percepciones y actitudes en relación con la alimentación y el cuerpo $y$, en definitiva, la manera de pensar y racionalizar la alimentación ${ }^{(5,6,7,8,9,10,11)}$. Al dejar de lado la mayor parte de los factores socioculturales que condicionan los comportamientos alimentarios, se desvirtúan los diagnósticos de las políticas educativas-sanitarias, disminuyendo así la probabilidad de su eficacia.

La alimentación es más que nutrición. A través del acto de alimentarnos, nos nutrimos. Además, mediante la alimentación, también nos comunicamos, expresamos y relacionamos. No comemos todo lo que es biológicamente comestible y esto significa que factores económicos, políticos, sociales e ideológicos condicionan nuestras elecciones alimentarias, así como las posibilidades de acceso y disponibilidad de los alimentos ${ }^{(12)}$. De la misma manera, estudiando los comportamientos alimentarios de la población, Ilegamos a entender mejor sus formas de vida ${ }^{(13)}$.

Marcel Mauss ya describía la alimentación como un "hecho social total"(14) que, por lo tanto, está cargado de la misma diversidad y complejidad que cualquier otro fenómeno social y como tal se debería abordar. $\mathrm{Y}$ es precisamente a esta diversidad y complejidad a las que prestamos especial atención en este artículo, es decir, cómo abordarlas, dado que una de las principales características de nuestra sociedad actual es la multiplicidad de formas de pensar, sentir y hacer la alimentación (funcionales, terapéuticas, ecológicas, locales y de proximidad, sostenibles, responsables, hedonistas, restrictivas, autónomas, solidarias) que, a la vez, son representativas de la gran variedad de maneras de pensar y hacer el mundo y que, en sus múltiples formas y niveles de interacción desembocan, a menudo, en controversias y conflictos no siempre fáciles de resolver.

En este sentido, Francesc Muñoz ${ }^{(15)}$ señala que cuando a la diversidad se le suma la complejidad, inevitablemente surge el conflicto y este, en términos alimentarios, está emergiendo de distintas formas en nuestra sociedad actual. Tenemos, por un lado, conflictos y controversias que tienen que ver con las elecciones alimentarias y con un contexto de abundancia sin precedentes. En este sentido, hoy más que nunca, parece que comer en función del gusto, las preferencias, el placer, la estética o los recursos económicos, sin que ninguno de estos criterios se contradiga, no es fácil y pone al comensal cotidiano en una difícil tesitura ${ }^{(12)}$ sobre la que conviene indagar para poder educar al respeto.

Asimismo, en los últimos años, se ha añadido otra controversia/conflicto alimentario que hace tambalear los fundamentos y principios democráticos de nuestro Estado de Bienestar: nos referimos al aumento de la inseguridad alimentaria en cuestión de acceso y disponibilidad de alimentos. Al respecto, los datos apuntan a que un tercio de los niños y las niñas del Estado español viven en riesgo 
de exclusión social y un 30\% están en riesgo nutricional ${ }^{(16)}$. Puede ser que sea el momento de que las políticas de educación nutricional no se planteen únicamente el reto de enseñar a escoger los alimentos, sino también el de la disponibilidad y el acceso. Parece absurdo pretender enseñar a elegir sin tener en cuenta las desigualdades que dificultan el acceso a los alimentos.

Toda esta complejidad alimentaria nos conduce a diversas cuestiones: ¿cuáles son los factores socioculturales y las desigualdades que condicionan ahora mismo las elecciones y el acceso a los alimentos de nuestros niños y niñas?; ¿qué dificultades o conflictos están emergiendo al respecto? $Y$, en consecuencia, ¿los mensajes educativos deben referirse a los nutrientes o los alimentos y sus formas de producción, distribución, comercialización y consumo?; ¿cómo hacer eficaz y responsable a la educación alimentaria en una sociedad de estas características y cómo evaluarla, sobre qué criterios?

A todo esto, se le añade el problema de que la mayoría de las políticas y actuaciones en educación nutricional ignoran a menudo que el aprendizaje alimentario no es un proceso que conste ni dependa únicamente de la información nutricional que se es capaz de adquirir, sino que está enmarcado dentro del proceso de educación y socialización infantil en su sentido más amplio ${ }^{(17)}$. Educar es más que informar o instruir, se trata de formar, de desarrollar las facultades de las personas de acuerdo a la cultura y sociedad para que las personas sean capaces de convivir de la manera más autónoma y responsable posible.

Por todas estas razones, interconectando teorías del conflicto social, de la antropología médica y los resultados de diversas investigaciones realizadas por nuestro grupo de investigación (el Observatorio de la Alimentación de la Universidad de Barcelona), este artículo pretende reflexionar sobre cómo la alimentación puede ser una herramienta para la educación y la transformación alimentaria, pero también social. En este sentido, proponemos un nuevo enfoque teórico-metodológico sobre educación alimentaria en el que la alimentación va más allá de la perspectiva nutricional e individualista, incorporando también una perspectiva política, económica, sociocultural y participativa que nos acerca a una comprensión innovadora del fenómeno: la alimentación como herramienta de análisis y diagnóstico de realidades socioalimentarias, pero también de intervención y cambio socioeducativo. En este sentido, apostamos por una educación alimentaria en la que la alimentación sea la finalidad (educar para aprender a comer), pero también un vehículo para educar en el sentido de ayudar a formar a niños y niñas para que sean capaces de resolver conflictos (personales y/o colectivos) e integrarse a su contexto social y alimentario de la manera más autónoma, crítica y responsable posible. Pensamos que, de este modo, fomentamos sociedades más saludables en términos globales, es decir, no solamente desde lo nutricional sino también desde lo social: desde el diálogo, el respeto, la igualdad y la cohesión social.

Parte de los resultados presentados en este estudio son producto de diversas investigaciones, entre ellas: "La alimentación y sus circunstancias: placer, conveniencia y salud

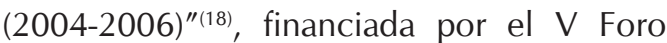
Internacional de la Alimentación; "Aprender a comer: procesos de socialización y trastornos del comportamiento alimentario"(19); y "Comer en la escuela y sus circunstancias: aprendizaje, cultura y salud (2009-2012)", financiadas por el Ministerio de Ciencia e Innovación de España (CSO2009-08741).

\section{MATERIAL Y MÉTODOS}

El artículo es fruto de diversos proyectos de investigación realizados, desde 2004 hasta la actualidad, en el Observatorio de la Alimentación (Universidad de Barcelona) y financiados, como se ha expuesto en el anterior apartado, por diversas entidades de reconocido prestigio nacional e internacional.

Las investigaciones han sido cualitativas, de base etnográfica y comparativa. Se han basado en la realización de trabajo de campo 
en ocho centros educativos de Cataluña (Tarragona y Barcelona) con diferente caracterización sociocultural según la situación sociodemográfica del colegio (centro de la ciudad y barrios de la periferia), las características ideológicas en términos de religiosidad o laicidad, y el carácter público o concertado (semipúblico).

Además de la consulta documental y bibliográfica, se ha llevado a cabo observación no participante en los diferentes espacios de los centros educativos implicados en la comensalidad de los menores (pasillos, patio, entrada y salida del colegio y comedor escolar), entrevistas semiestructuradas y en profundidad y grupos de discusión. Se han realizado 117 entrevistas semiestructuradas a niños y niñas de entre 6 y 16 años sobre sus actitudes y hábitos alimentarios. También se han realizados más de veinte entrevistas en profundidad a niños y niñas de 6 a 16 años con la intención de profundizar en los porqués ${ }^{(20)}$ de sus prácticas y actitudes alimentarias. Se realizaron, asimismo, cinco grupos de discusión con diferentes agentes participantes de la educación alimentaria infantil: tres grupos de discusión con monitores/as y personal de comedor escolar, un grupo de discusión con profesores y un grupo de discusión con padres y madres.

En el caso de los participantes menores de edad, se obtuvo el consentimiento informado de los padres a través de los centros educativos, y sus maestros y maestras les informaron acerca de los objetivos y el alcance de la información. Las personas participantes en el estudio fueron informadas de los objetivos y métodos de la investigación, se obtuvo el consentimiento informado de cada una de ellas. Se garantizó el anonimato y la confidencialidad de los datos de todos los participantes durante las distintas etapas de proceso proceso.

Las entrevistas fueron transcritas literalmente, revisadas y tratadas de forma confidencial. Posteriormente, los datos fueron organizados en una unidad hermenéutica, codificados y explotados de forma sistemática y exhaustiva a través del programa de análisis cualitativo MAXQDA. Este programa ha permitido efectuar un análisis de contenido temático del material empírico recogido mediante método inductivo de relectura y codificación, y generar un marco explicativo con relación al objetivo de la investigación.

\section{ALGUNAS PREMISAS SOBRE CONFLICTO SOCIAL}

La diversidad y multiculturalidad de Europa es un hecho constatado y en continuo auge. Andreas Kaplan ${ }^{(21)}$ describe Europa como un continente que abraza una máxima diversidad cultural en una superficie geográfica mínima. Esta diversidad se plasma en los diferentes ámbitos de la vida cotidiana como pueden ser el arte, la cultura, la religión, la educación, la alimentación, etc. Sin embargo, delante de esta enriquecedora diversidad, también están emergiendo (nuevas) formas de discriminación y desigualdad que generan, a su vez, conflictos de orden también diverso.

En este sentido, muchos expertos coinciden en el hecho de que cuando a los contextos diversos se les suma la complejidad, inevitablemente surge el conflicto ${ }^{(15)}$. Este suele emerger como fruto de interacciones sociales que generan desacuerdos, desigualdad, incluso de situaciones en las que no se reconoce la diferencia como un derecho personal o social.

En este sentido, el orden y la integración social han sido, y continúan siendo, los debates de fondo en la historia de la teoría social. Por este motivo, el conflicto social ha ocupado un lugar central en la reflexión dentro de disciplinas como la filosofía, la sociología o la antropología, y lo tomamos también como base en este trabajo para articular nuestra propuesta final.

Por una parte, consideramos el conflicto desde posturas como las de Simmel ${ }^{(22)}$, Giner $^{(23)}$ o Tejerina ${ }^{(24)}$, que lo interpretan como un fenómeno siempre presente en cualquier sociedad, que cambia y, a la vez, provoca cambios sociales. En este sentido, el conflicto social se contempla como paradójicamente necesario para promover la 
transformación social e ir hacia sociedades más cohesionadas. Se entiende, asimismo, que todo conflicto social forma parte de una realidad social más amplia; por lo tanto, puede operar como un mecanismo de producción de la sociedad y como una forma de socialización $^{(24)}$. También se apunta a que el estudio del cambio social ha desarrollado esquemas y teorías basadas, precisamente, en el conflicto social(23). De ambas reflexiones se desprende la idea de que los cambios sociales implican a menudo conflictos previos que, a su vez, promueven cambios sociales porque permiten disolver las divergencias para llegar a nuevos modelos de integración social. En este sentido, el conflicto social se consideraría como un fenómeno integrador, favorecedor del cosmopolitismo, el respeto y la convivencia, que no tiene por qué entenderse necesariamente como un factor de disolución social(22).

La resolución del conflicto social precisa, no obstante, de un contexto de diálogo sociocultural para ser llevada a cabo. En este sentido, diferentes autores ${ }^{(25,26,27,28)}$ señalan que todo conflicto implica necesariamente un proceso de comunicación verbal, escrito o corporal en el que se dan incompatibilidades de intereses por parte de dos o más personas que interactúan. Asimismo, apuntan que, para negociar y resolver un conflicto, ambas partes tienen que perder y ganar algo. En este proceso, además, las personas, grupos o comunidades afectadas deben relacionarse, interaccionar y dialogar de forma que se puedan conocer, entender, respetar y negociar los motivos por los cuales cada una de ellas defiende su postura.

\section{Conflictos alimentarios en nuestra sociedad actual}

Según señala la Food and Agriculture Organization $(\mathrm{FAO})^{(29)}$, el derecho a una alimentación adecuada es un derecho humano internacional que existe desde hace ya tiempo y al que se han comprometido muchos países, pero que está lejos de haberse cumplido. En las últimas décadas, diferentes países han desarrollado e implementado reformas constitucionales, leyes nacionales, estrategias, políticas y programas que tienen como objetivo el cumplimiento del derecho a la alimentación para todas las personas. A nivel mundial, el derecho a una alimentación adecuada ha sido un derecho humano legalmente vinculante en el derecho internacional desde hace más de treinta cinco años $y$, desde entonces, se han otorgado garantías jurídicas adicionales a grupos específicos, como las mujeres, los niños y las personas con discapacidades. En 2004, los Estados miembros de la FAO aprobaron por consenso las "Directrices voluntarias en apoyo de la realización progresiva del derecho a una alimentación adecuada en el contexto de la seguridad alimentaria nacional", que ofrecen orientación práctica sobre la forma de aplicar el derecho a una alimentación adecuada en una amplia gama de áreas de políticas y programas. Más recientemente, las "Directrices voluntarias sobre la gobernanza responsable de la tenencia de la tierra, la pesca y los bosques en el contexto de la seguridad alimentaria nacional" o el "Reto Hambre Cero" de las Naciones Unidas, priorizan el cumplimiento del derecho a la alimentación ${ }^{(29)}$.

Sin embargo, según esta misma organización, hay más de 793 millones de personas que se van a dormir con hambre todas las noches. En España, un tercio de los niños y niñas viven en riesgo de exclusión social, un $30 \%$ están en riesgo nutricional y uno de cada cuatro no come fruta ni verdura diariamente ${ }^{(16)}$. También ha aumentado el número de niños y niñas que no almuerzan antes de ir al colegio, así como las iniciativas sociales y no gubernamentales que reparten comida en las escuelas. Sin embargo, y debido a su carácter jurídicamente vinculante, la realización del derecho a una alimentación adecuada no se debería cumplir a través de las acciones asistencialistas o la beneficencia ${ }^{(29)}$. El derecho a la alimentación es un derecho humano de cada mujer, hombre, niña y niño que ha de cumplirse con acciones apropiadas de los gobiernos y los actores no estatales. No obstante, en el Estado español, muchas asociaciones y agrupaciones del tercer sector se 
han visto abocadas a formas de intervención asistencialista debido a los recortes presupuestarios realizados por el gobierno a raíz de la crisis socioeconómica iniciada en 2008. Este fenómeno ha derivado en un incremento importante de las desigualdades en términos de acceso y disponibilidad de alimentos, así como en el aumento de los conflictos entre familias y comunidades por el acceso a las ayudas sociales (becas de comedor escolar, por ejemplo).

Además, los conflictos alimentarios tienen otra cara más sutil, que va más allá de las desigualdades macroeconómicas en el acceso y disponibilidad de los alimentos. Los denominaremos microconflictos alimentarios, siguiendo el esquema micro-macro de las teorías sociológicas. A diferencia de los anteriores (que podríamos denominar macroconflictos alimentarios), los microconflictos alimentarios serían aquellos que se caracterizan por la no homogeneidad, la lógica de la separación y del intercambio; en los que prevalece más el individuo que el grupo (aunque sin desvincularse de él ni de la estructura social que lo determina), y que tendrían que ver con la dimensión más personal, interna y simbólica de las elecciones alimentarias. Nos referimos, más concretamente, a las numerosas contradicciones que sitúan a nuestro comensal cotidiano en una situación difícil y/o conflictiva a la hora de elegir qué y cómo comer en un espacio socioalimentario como el nuestro, caracterizado por una abundancia sin precedentes, pero también mercantilizado y biopolitizado ${ }^{(30)}$. Cuando hablamos de biopolitizado nos referimos a la perspectiva foulcaultiana ${ }^{(30)}$ sobre el control de los gobiernos y el poder sobre los cuerpos de la población a través de la alimentación: laboratorios farmacéuticos y grandes empresas que intentan monopolizar la producción alimentaria (por ejemplo, los transgénicos); así como a la mercantilización de nuestros cuerpos a través de una gran oferta de alimentos con finalidades diversas: estéticas, de salud, placer, confort, etc. Desde pequeños, a los niños y las niñas se les empieza a transmitir responsabilidades. Desde este momento, se pueden ir presentando conflictos personales relacionadas con las exigencias y/o expectativas que van asumiendo e interiorizando desde el orden socioeducativo más amplio (familia, grupo de amigos y amigas, escuela, medios de comunicación, etc.) y que tienen que ver, en el caso concreto de la alimentación, con aspectos tan diversos como: qué alimentos se consideran buenos o malos para la salud; qué alimentos y cómo comer para alcanzar una imagen estética y corporal ideal; qué y cómo comer para aprender a relacionarse con los demás y en los diferentes contextos y ámbitos de la vida personal-laboral-familiar, etc. En definitiva, qué y cómo comer para alcanzar el éxito social.

Ahora mismo, en nuestra sociedad actual tenemos desde alimentos fáciles de cocinar que nos sirven para ahorrar tiempo en una sociedad marcada por las prisas, el estrés cotidiano y la productividad, a productos que vienen a paliar, precisamente, los efectos de todo eso y que fomentan el placer y la comodidad. Asimismo, junto a la oferta homogeneizadora tenemos iniciativas privadas y sociales que buscan dar al alimento y a su consumidor identidad y distinción. A su vez, también tenemos alimentos funcionales e iniciativas ecológicas que pretenden mejorar nuestro estado de salud (la ciudad de Barcelona, por ejemplo, se ha declarado ciudad veg-friendly). No obstante, este amplio abanico de posibilidades comporta a menudo contradicciones como la promoción de productos estéticos que rebaten el discurso médico-nutricional y de salud ${ }^{(12,13)}$. De hecho, diversos estudios nuestros $^{(18,19)}$ han puesto de manifiesto las controversias en las que muchos padres se encuentran a la hora de educar alimentariamente a sus hijos e hijas:

Es que lo cierto es que la misma sociedad es una contradicción... Ves los anuncios de la TV y te ponen que si este o el otro producto son buenos para la salud de tu hijo, que si el yogur tal con lactobacilus o cosas de estas que ni siquiera sabes lo que quieren decir, pero que se supone que son buenas para la salud. Y luego resulta que el médico te dice que de esas cosas no hagas caso. Luego, por ejemplo, 
con el chocolate, ¿vale? Te dicen que está enriquecido con esto o lo otro, ¿vale? Y, además, que un poco de chocolate no es malo, que hay que comer de todo... Entonces... cuando viene mi niño con un Kinder de estos, que se supone que no es muy bueno para la salud, pero que a él le gusta mucho, pero que si come un poco no pasa nada... ¿Qué le digo? ¿Se lo prohíbo o no? ¿Dónde está el límite de lo bueno o lo malo? (Grupo de discusión, padres-madres).

En definitiva, parece que comer en función del gusto, las preferencias, la salud, la estética o los recursos económicos, sin que ninguno de estos criterios se contradiga, no es fácil y pone al comensal cotidiano en una difícil tesitura, a menudo generadora de controversias y conflictos, sobre la que pensamos que es necesario intervenir, educar.

\section{Alimentación y comunicación, alimentación y educación: la alimentación en la resolución de conflictos}

Es opinión unánime, al menos dentro de las disciplinas que estudian la alimentación desde una perspectiva social y humana, que la alimentación es más que nutrición. A través del acto de alimentarnos, nos nutrimos, pero además, mediante la alimentación también nos comunicamos, expresamos y relacionamos. No comemos todo lo que es biológicamente comestible y esto significa que factores económicos, políticos, sociales e ideológicos condicionan nuestras elecciones alimentarias, así como las posibilidades de acceso y disponibilidad de los alimentos ${ }^{(12)}$. De la misma manera, estudiando los comportamientos alimentarios de la población, llegamos a entender mejor sus formas de vida $^{(13)}$. Mauss ${ }^{(14)}$ ya describía la alimentación como un "hecho social total" y Douglas señalaba que los principios de selección que guían al ser humano en la elección de sus recursos alimenticios no son de orden fisiológico, sino cultural|(31).
Según qué comemos y cómo lo comemos se nos puede identificar individual o colectivamente, según seamos hombres o mujeres (género), nacidos en un lugar u otro (etnia), pertenecientes a una clase social o a otra, etc. Por eso, podemos afirmar que la alimentación es un potente sistema de comunicación que emite significados de la sociedad en la que se inscribe: lo que comemos depende de lo que somos (de las formas de vivir - pensar y hacer- de un pueblo o cultura), pero también podemos llegar a conocer cómo somos, conociendo lo que comemos, ya que cada comportamiento o actitud alimentaria -personal o colectiva- tiene un significado concreto que solamente toma sentido dentro de la sociedad y la cultura en la que se inserta.

En general, la cultura actúa estableciendo normas determinadas según cada sociedad y conforma así formas de organización y de vida, en definitiva, formas determinadas de pensar y hacer. Por lo que refiere al comportamiento alimentario, la cultura establece normas relativas a la composición y a la estructura de las ingestas, a las técnicas culinarias, a los horarios de las comidas, a los comensales, a los lugares de cada comida, a la selección de los alimentos y a la manera de mezclarlos y manipularlos, al tratamiento de conservación, a las formas de servir y de comer, a la distribución de las comidas, a la idoneidad o no de los alimentos (los que son más o menos saludables, buenos o malos según para qué aspectos). Del mismo modo, la cultura marca los modales que socialmente se entienden como correctos para comer: si se tiene que comenzar con un tipo $\mathrm{u}$ otro de ingesta, los calendarios alimentarios según las ocasiones festivas, así como los modelos alimentarios según género, edad o clase social ${ }^{(13)}$. De hecho, el género es fundamental para comprender y explicar tanto las diferentes concepciones y prácticas aprehendidas en relación con la alimentación, el cuerpo y la salud, como la diferente y desigual incidencia de determinadas problemáticas alimentarias -como los trastornos alimentarios- en hombres y mujeres. En uno de nuestros estudios ${ }^{(9)}$ comprobamos que el significado que va adquiriendo el comer y el 
no comer para los niños y niñas es distinto y desigual en tanto que también lo es su contexto socializador, es decir, la manera como interaccionan en y con el medio familiar, escolar, grupo de iguales, etc. En este sentido, observamos que las mujeres se socializan más en un modelo en el que la comida es utilizada como herramienta de control corporal y de expresión de malestares, mientras que los hombres suelen expresarse y controlar su cuerpo a través del ejercicio físico porque también suelen socializar más en la práctica deportiva y en las actividades de control del medio $^{(19)}$ :

Hacía baloncesto de pequeña, pero ahora ya no hago deporte, casi nada. No sé, mis amigas también lo han dejado de hacer, no es una cosa que la veas importante para ser alguien en la vida... no sé. Yo, si quiero adelgazar, dejo de comer (Julia, 16 años).

Prefiero hacer deporte y machacarme jugando a fútbol y corriendo... iQue dejar de comer! Yo no entiendo cómo algunas de mis compañeras dejan de almorzar al patio... yo necesito mi bocata y si hace falta, luego lo quemo todo corriendo (Juan, 16 años).

Por otra parte, todas estas normas no son fruto del arbitrio, sino tributarias de los condicionamientos sociales, culturales, económicos, políticos e ideológicos de cada sociedad. En este sentido, toda la cadena alimentaria -desde la producción hasta el consumo de alimentos- se inserta en una serie de procesos ecológicos, tecnológicos, económicos, políticos e ideológicos complejos que determinan, en buena medida, la dinámica y la lógica de los comportamientos alimentarios. Por lo tanto, cuando hablamos de cultura alimentaria nos referimos al conjunto de actividades condicionadas por los factores anteriormente mencionados, establecidas por un grupo humano y cuya finalidad es obtener los alimentos necesarios para subsistir. Esto incluye, además, procesos como el aprovisionamiento, la producción, la distribución, el almacenaje, la conservación, la preparación y el consumo, así como un conjunto de reglas, normas, creencias y conocimientos que organizan y orientan las formas de alimentarse de una determinada sociedad y de las personas y sujetos que la conforman $^{(13)}$.

Por otra parte, la cultura establece una regularidad (normas) en la alimentación de cada sociedad y eso también confiere especificidad, en la medida que no hay dos culturas iguales y, por tanto, tampoco hay dos culturas alimentarias (ni cocinas) iguales. Sin embargo, las culturas alimentarias no son estáticas, cambian conforme las diversas interacciones sociales de los sujetos que las conforman. Por este motivo, y bajo el paraguas del construccionismo social y de la teoría social de la práctica, entendemos que cada sujeto aprehende y se socializa en y de su respectiva cultura alimentaria, asumiendo e interiorizando las normas y valores socioalimentarios de su sociedad. Sin embargo, no debemos entender este proceso de socialización como algo estático ya que, a pesar de que el género, la edad, la clase social o la etnia -entre otras muchas variables socioculturales- determinan nuestras elecciones alimentarias, la socialización y el aprendizaje alimentario debe analizarse, también, desde los mecanismos de negociación y reinterpretación que cada sujeto hace sobre estas normas sociales, (re)produciendo y/o transformando, asimismo, el propio sistema social.

Por lo tanto, si como acabamos de ver cada cultura alimentaria es fruto de un contexto social, político, ideológico e histórico determinado, también podemos decir que la alimentación es un sistema de comunicación poderoso que transmite información sobre las características de cada persona, grupo o comunidad. A través de lo que comemos transmitimos lo que somos. Cada plato, cada alimento, cada ingrediente, la manera como lo combinamos, las categorizaciones de los diferentes alimentos, los principios de exclusión y de asociación entre uno y otro alimento, las prescripciones y las prohibiciones tradicionales o religiosas, los rituales de la mesa y de la cocina (recetas), los diferentes 
usos de los alimentos, el orden en que se ingieren, la composición, la hora y el número de ingestas diarias, etc., están enviando información sobre una sociedad y su población ${ }^{(13)}$. Asimismo, las diferentes maneras de alimentarse pueden constituir un medio para identificarse, darse a conocer o reafirmarse frente al otro, afirmar el propio estatus, adquirir o no prestigio, promocionarse socialmente, manifestar emociones, aceptación o rechazo, etc.

En definitiva, la alimentación constituye una de las características culturales que más fácilmente perviven en el contacto con otras culturas y se alza con fuerza como signo que marca y transmite identidad ${ }^{(32,33,34)}$. Por lo tanto, dada su carga de significados, la alimentación también puede alzarse como una poderosa herramienta para el diálogo $\mathrm{y}$, consecuentemente, para la resolución de conflictos.

\section{DISCUSIÓN FINAL}

\section{Educar la alimentación o la alimentación como forma de aprendizaje: propuestas para una educación alimentaria inclusiva}

En estas páginas, hemos podido ver la gran diversidad y complejidad que envuelve al hecho alimentario en nuestra sociedad actual. Existen diferentes formas de pensar, sentir y hacer la alimentación que a la vez son representativas de las diversas maneras de pensar y hacer en el mundo y que, en sus múltiples formas y niveles de interacción social desembocan, a menudo, en controversias y conflictos (macroconflictos y microconflictos alimentarios) no siempre fáciles de resolver. Asimismo, hemos visto que los conflictos y controversias alimentarias tienen que ver con las desigualdades en el acceso y la disponibilidad de los alimentos, pero también con las sutilezas de una biopolítica ${ }^{(30)}$ y un mercado que dificultan las elecciones alimentarias.

En este sentido, el abordaje de las problemáticas alimentarias emergentes desde las teorías del conflicto social nos ha permitido entenderlas y comprenderlas mejor desde una panorámica social, pero, además, nos ha ofrecido pistas sobre sus posibilidades a la hora de resolverse o solucionarse (resolución de conflictos). Por un lado, nos ha permitido entender el conflicto social como un fenómeno siempre presente en cualquier sociedad y necesario para promover la transformación social e ir hacia sociedades más cohesionadas ${ }^{(22,23,24)}$. Por otra parte, hemos visto que en la resolución de conflictos el diálogo y la comunicación son fundamentales ${ }^{26,27,28)}$. Asimismo, hemos podido ver que la alimentación es un sistema de comunicación poderoso, cargado de significados sobre nuestras identidades individuales y colectivas.

Por lo tanto, si como acabamos de señalar, el conflicto social (y también alimentario) es necesario para el cambio y la transformación social (y también socioalimentaria); la comunicación y el diálogo son herramientas para la resolución de los conflictos; y la alimentación es un potente sistema de comunicación. Proponemos un nuevo enfoque sobre la educación alimentaria que aproveche el potencial dialógico y socioeducativo de la alimentación para resolver problemáticas y conflictos, así como para la transformación social y el fomento de la cohesión social.

En este sentido, nuestra propuesta apunta a dos partes del proceso de cualquier intervención social, en este caso socioeducativa: a) la primera, que es el estudio y diagnóstico de las problemáticas, en este caso, problemáticas socioalimentarias; y b) el diseño metodológico que utilizamos para intervenir sobre estas problemáticas y transformarlas (erradicarlas, mejorarlas, etc.).

Con respecto a la primera fase, centrada en el estudio-diagnóstico de las problemáticas y conflictos alimentarios, proponemos modelos de diagnósticos socioalimentarios más inclusivos que vayan más allá del estudio de lo nutricional como único desencadenante de salud, ya que la alimentación es más que nutrición y la salud tiene que ver con el bienestar físico, pero también psicosocial. En este sentido, hemos visto que la mayoría de los planes, programas, proyectos o campañas sobre educación alimentaria se centran en la dimensión nutricional de la alimentación, 
dejando de lado o en segundo término los factores socioculturales (sociales, políticos, económicos, históricos e ideológicos) que son, precisamente, los que condicionan las elecciones alimentarias, así como el acceso y la disponibilidad de los alimentos ${ }^{(12,6,7,11)}$. Por eso, necesitamos métodos de investigacióndiagnóstico que nos permitan ir más allá de los alimentos y de sus componentes nutricionales, para estudiar también los procesos de enseñanza-aprendizaje en los que se insertan. Nos referimos a indagar sobre qué comen los niños y niñas, pero también sobre dónde lo comen, cuándo, cómo, con quién y por qué lo comen. Se trata, por lo tanto, de profundizar en la diversidad y complejidad de las prácticas y actitudes alimentarias para cada persona, grupo o comunidad y en las diferentes situaciones, circunstancias y contextos socioeducativos en los que se enmarcan. Esto implica, además, pensar en técnicas concretas de recogida y análisis de información cualitativas, menos simplificadoras, que consigan conocer la parte nutricional de los alimentos en relación con el contexto sociocultural de su producción, distribución, elaboración y consumo. Solo así podremos desentrañar las posibles desigualdades que están determinando las diferentes prácticas y actitudes alimentarias, así como las causas de los conflictos personales o sociales en los que están desembocando.

Con respecto al segundo paso de la intervención socioeducativa, centrada en el diseño y elaboración de la propuesta metodológica de intervención socioeducativa, proponemos utilizar la alimentación como herramienta para la educación, en este caso, para educar en y para la convivencia y la cohesión social. En este caso, nos referimos a la importancia de enseñar a alimentarse, pero también, a utilizar la alimentación como vehículo socioeducativo. Dado el potencial dialógico de la alimentación, pensamos en las posibilidades que puede ofrecer una educación alimentaria generadora de espacios de diálogo en los que los propios niños y niñas puedan intercambiar sus experiencias alimentarias vinculadas a las situaciones personales y colectivas concretas que las explican: situaciones económicas, históricas, políticas, ideológicas, etc. A través de motivos alimentarios diversos (festividades, cumpleaños, comida escolar, comida familiar, picnics, excursiones, viajes, etc.), podemos generar espacios de convivencia y reflexión en torno a la producción, la distribución, la elaboración o el consumo alimentario, de modo que los niños y niñas desarrollen sus capacidades críticas y puedan convertirse, así, en protagonistas directos de su propio proceso de aprendizaje alimentario, así como de los cambios y transformaciones que este aprendizaje autónomo implique en el marco más amplio de su/nuestra sociedad.

En definitiva, el análisis cualitativo de la alimentación nos permite estudiar y entender los porqués de los comportamientos alimentarios, así como las experiencias individuales y colectivas que les dan sentido. Del mismo modo, la alimentación puede ser aprovechada para educar en, desde y para lo social. Puede ser, por lo tanto, una magnífica herramienta de estímulo de la reflexión y la crítica $y$, en consecuencia, de empoderamiento individual y social de personas, grupos y comunidades que buscan alcanzar y hacer plausibles sus derechos sociales y de salud. En este sentido, la alimentación nos permite detectar y desentrañar las causas de muchas desigualdades y conflictos personales y sociales, así como intervenir de forma socioeducativa para erradicarlos o, en todo caso, favorecer políticas de educación alimentaria más inclusivas que promocionen sociedades más saludables en términos globales, es decir, no solamente nutricionales sino también en términos sociales de integración, igualdad y cohesión social. 


\section{AGRADECIMIENTOS}

Parte de los resultados presentados en este artículo son producto de diversas investigaciones: "La alimentación y sus circunstancias: placer, conveniencia y salud (2004-2006)", realizada por el Observatorio de la Alimentación y financiada por el V Foro Internacional de la Alimentación; la tesis doctoral "Aprender a comer: procesos de socialización y trastornos del comportamiento alimentario" (Zafra, 2007); y "Comer en la escuela y sus circunstancias: aprendizaje, cultura y salud" (2009-2012) del Observatorio de la Alimentación (Universidad de Barcelona) y financiada por el Ministerio de Ciencia e Innovación (CSO200908741). La autora agradece la colaboración de los diferentes miembros de cada uno de los proyectos y expresa su especial gratitud a todos los informantes que participaron en las investigaciones por compartir su tiempo y sus experiencias.

\section{REFERENCIAS BIBLIOGRÁFICAS}

1. Organización Mundial de la Salud. Estrategia Mundial sobre Régimen Alimentario, Actividad Física y Salud [Internet]. 2014 [citado 11 may 2016]. Disponible en: https://tinyurl.com/y9tecwm9.

2. Comisión de las Comunidades Europeas. Libro Blanco: Estrategia europea sobre problemas de salud relacionados con la alimentación, el sobrepeso y la obesidad [Internet]. Bruselas; 2007 [citado 27 abr 2016]. Disponible en: https://tinyurl. com/yadekrzp.

3. Agencia Española de Seguridad Alimentaria. Estrategia NAOS: Estrategia para la nutrición, actividad física y prevención de la obesidad [Internet]. Madrid: Ministerio de Sanidad y Consumo; 2005 [citado 5 may 2016]. Disponible en: https://tinyurl. com/y9xqsde5.

4. Agencia Española de Seguridad Alimentaria y Nutrición. Plan Cuídate+2012 [Internet]. Madrid: Ministerio de Sanidad, Servicios Sociales e Igualdad; 2012 [citado 2 oct 2016]. Disponible en: http://www.plancuidatemas.es

5. Tibbs T, Haire-Joshu D, Schechtman K, Brownson $\mathrm{R}$, Nanney M, Houston $\mathrm{CH}$, Auslander W. The relationship between parental modeling, eating patterns, and dietary intake among African-american parents. Journal of the American Dietetic Association. 2001;101(5):535-541.

6. Rozin P, Hormes J. Food preferences and aversions. In: Shweder RA, Bidell TR, Dailey AC, Dixon SD, Miller PJ, Modell J. The Child: An Encyclopedic Companion. Chicago: Univer; 2009.
7. Zafra E. Comer en la escuela: el modelo de educación alimentaria en el comedor escolar. Trabajo Social y Salud. 2005;(51):361-382.

8. Zafra E. ¿Re-educar para educar? La (re)educación alimentaria en el comedor escolar: dificultades en el aprendizaje de la dieta equilibrada. En: Martorell MA, Comelles JM, Bernal M. Antropología y enfermería. Tarragona: Publicacions URV; 2010.

9. Zafra E. La transversalidad de los procesos de socialización en los comportamientos alimentarios de niños y niñas. Zainak. 2011;(34):33-60.

10. Atie B, Contreras J, Zafra E. Comer en la escuela y sus circunstancias: aprendizaje, cultura y salud. Zainak. 2011;(34):61-74.

11. Contreras J, Gracia M, Atie B, Pareja SL, Zafra E. Comer en la escuela: una aproximación etnográfica. En: Martínez JR. Nutrición y alimentación en el ámbito escolar. Madrid: ERGON; 2012.

12. Fischler C. El (h)omnívoro. Barcelona: Anagrama; 1995.

13. Contreras J, Gracia M. Alimentación y cultura: Perspectivas antropológicas. Barcelona: Ariel; 2005.

14. Mauss M. Sociologie et Anthropologie. Paris: PUF; 1950

15. Muñoz F. Urbanalización: paisajes comunes, lugares globales. Barcelona: Editorial Gustavo Gili; 2008.

16. Save the Children [Internet]. [Citado $4 \mathrm{abr}$ 2015]. Disponible en: https://www.savethechildren.es

17. Del Valle T. Mujer y nuevas socializaciones: su relación con el poder y el cambio. Kobie. 1992;(6):5-15.

18. V Foro Internacional de la Alimentación. La alimentación y sus circunstancias: placer, conveniencia y salud. Barcelona: V Foro Internacional de la Alimentación; 2004.

19. Zafra E. Aprender a comer: procesos de socialización y trastornos del comportamiento alimentario [Tesis doctoral]. Barcelona: Departament d'Antropologia Cultural i Història d'Amèrica i d'Àfrica, Universitat de Barcelona; 2008.

20. Tylor SJ, Bodgan R. Introducción a los métodos cualitativos de investigación. Buenos Aires: Paidós; 1992. 
21. Kaplan A. European Management and European Business Schools: Insights from the history of business schools. European Management Journal. 2014;32(4):529-534.

22. Simmel G. Sociología: estudios sobre las formas de socialización. Madrid: Revista de Occidente; 1977.

23. Giner S. El progreso de la conciencia sociológica. Barcelona: Península; 1978.

24. Tejerina B. Las teorías sociológicas del conflicto social: Algunas dimensiones analíticas a partir de K Marx y G Simmel. REIS. 1991;(55):47-64.

25. Aron R. Paz y guerra entre las naciones. Madrid: Revista de Occidente; 1963.

26. Robbins SP. Comportamiento organitzacional: conceptos, controversias y aplicaciones. México: Prentice Hall; 1994.

27. Suárez M. Mediación: Conducción de disputas y técnicas. Buenos Aires: Plaza Edición; 1996.

28. Fisas V. Cultura de paz y gestión de conflictos. Barcelona: Icaria Ediciones; 2002.

29. Organización de las Naciones Unidas para la Alimentación y la Agricultura. Derecho a la ali- mentación [Internet] [citado 13 sep 2016]. Disponible en: https://tinyurl.com/y8sut54n.

30. Foucault M. Vigilar y castigar. Madrid: Siglo XXI Editores; 1992.

31. Douglas M. Las estructuras de lo culinario. En: Contreras J. Alimentación y cultura: Necesidades, gustos y costumbres. Barcelona: Publicaciones de la Universitat de Barcelona; 1995.

32. Bourdieu P. La distinción: Criterios y bases sociales del gusto. Madrid: Taurus; 1988.

33. Hubert A. Alimentation et santé: la science et I'imaginaire. Cahiers de Nutrition et de Diétetique. 2000;35(5):353-356.

34. Lacomba J. El Islam inmigrado: Transformaciones y adaptaciones de las prácticas culturales y religiosas. Madrid: MEC; 2001.

35. Rogoff B. Aprendices del pensamiento: El desarrollo cognitivo en el contexto social. Barcelona: Paidos; 1993.

36. Morton H. Becoming Tongan: An etnography of childhood. Honolulu: University of Hawai Press; 1996.

Recibido: 29 de octubre de 2016 | Versión final: 19 de enero de 2017 | Aprobado: 31 de enero de 2017

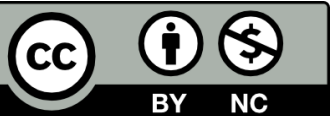

